\title{
Vector Unpacking as a Potential Barrier for Receptor-Mediated Polyplex Gene Delivery
}

\author{
David V. Schaffer, ${ }^{1}$ Nick A. Fidelman, ${ }^{1}$ Nily Dan, ${ }^{2}$ Douglas A. Lauffenburger ${ }^{1}$ \\ ${ }^{1}$ Department of Chemical Engineering and Biotechnology Process \\ Engineering Center, Massachusetts Institute of Technology, \\ Cambridge, Massachusetts \\ ${ }^{2}$ Department of Chemical Engineering, Drexel University, \\ Philadelphia, Pennsylvania
}

Received 30 June 1999; accepted 3 October 1999

\begin{abstract}
Ligand-conjugated polymer (polyplex) gene delivery vectors have strong potential as targeted, in vivo gene transfer vehicles; however, they are currently limited by low delivery efficiency. A number of barriers to polyplex-mediated delivery have been previously identified, including receptor binding, internalization, endosomal escape, and nuclear localization. However, based on understanding of viral gene delivery systems, yet another potential barrier may exist; a limited ability to unpackage the plasmid DNA cargo following localization to the nucleus. We have developed a model system that employs a cationic polymer linked to epidermal growth factor (EGF) as a ligand to target delivery of plasmid DNA encoding the green fluorescent protein to mouse fibroblasts bearing the EGF receptor. Using fluorescence microscopy to simultaneously trace both the plasmid and polymer during gene delivery in combination with an in vitro transcription assay, we provide evidence that plasmid unpackaging can indeed be a limiting step for gene expression for sufficiently large polymer constructs. Short-term expression is significantly enhanced by using short polycations that dissociate from DNA more rapidly both in vitro and in vivo. Finally, we describe a thermodynamic model that supports these data by showing that shorter polycations can have a higher probability of dissociating from DNA. This work demonstrates that vector unpackaging should be added to the list of barriers to receptor-mediated polyplex gene delivery, thus providing an additional design principle for targeted synthetic delivery vehicles. (c) 2000 John Wiley \& Sons, Inc. Biotechnol Bioeng 67: 598-606, 2000.
\end{abstract}

Keywords: synthetic; nonviral; gene; delivery; therapy; polymer

\section{INTRODUCTION}

Interest in gene therapy has soared in recent years due to its vast potential for treating a variety of diseases; however,

Correspondence to: D. Schaffer, Chemical Engineering Department, MC 1462, University of California at Berkeley, Berkeley, CA 94720-1462; Telephone: 510-643-5963; fax: 510-642-4778; e-mail: schaffer@cchem. berkeley.edu

Contract grant sponsors: Fannie and John Hertz Foundation; NSF Biotechnology Process Engineering Center (MIT), ND was partially supported by NSF-BES 0096004 significant progress must still be made before safe, reliably manufacturable gene delivery vehicles are developed, especially for targeting to specific cell types via receptormediated uptake. Although significant progress is being made on learning how to target the more common viral vehicles (Kovesdi et al., 1997), synthetic vehicles based on polymer-ligand conjugates are an attractive long-term solution because of their potential advantages in safety, manufacturability, nucleic acid cargo capacity, and designability (Paillard, 1998).

Molecular conjugate, or "polyplex" vehicles are generated by condensing nucleic acids with a cationic polymer that can be chemically crosslinked to a ligand in order to direct delivery to specific target cells. Research with conjugates has established their ability for targeted delivery to a variety of receptors and cells (Hart et al., 1995; Perales et al., 1997; Sosnowski et al., 1996; Wagner et al., 1994; Wu and $\mathrm{Wu}, 1987$; Ziady et al., 1997), the capacity to carry large nucleic acid constructs (Cotten et al., 1992), a potential for low toxicity and immunogenicity (Plank et al., 1996; Stankovics et al., 1994), and protection of their genetic cargo from nuclease digestion (Chiou et al., 1994). These safe vectors therefore mimic the ability of viruses to package DNA, bind to cells, and deliver their "genome" to the cell nucleus. Despite their promise, however, polyplex vectors are currently limited by low gene-delivery efficiency.

Improvements in efficiency can be achieved through a detailed examination of the mechanism of and identification of barriers to conjugate gene delivery. Previous work has demonstrated that binding to the cell surface (Schaffer and Lauffenburger, 1998), escape from the endosomal network (Cotten et al., 1992; Curiel et al., 1991; Plank et al., 1994), and translocation to the nucleus (Capecchi, 1980; Sebestyen et al., 1998) are rate-limiting steps for DNA transfer, and engineering a vector to overcome these barriers can yield significant gains in delivery. Here, we investigate whether the final step of the gene delivery process, unpackaging of the polyplex, can limit the efficiency of gene delivery and expression. The rationale for this work is that, although 
some viruses have evolved highly specific and concerted mechanisms for uncoating within the cell, a synthetic polycation may not release DNA with similar high efficiency. Some recent work (Godbey et al., 1999a, 1999b), in fact, has demonstrated interesting effects of polycation size properties that could possibly be explained by this mechanism.

Our results from a combination of approaches, including fluorescence microscopy, in vitro transcription, and gene expression, show that vector unpackaging can indeed be a limiting barrier to receptor-mediated synthetic conjugate gene delivery under some conditions, and that delivery efficiency can be enhanced significantly by using shorter polycations that release DNA more easily.

\section{MATERIALS AND METHODS}

\section{Materials}

Mouse EGF was obtained from Life Technologies. Proteins were iodinated with $\mathrm{Na}^{125} \mathrm{I}$ (New England Nuclear) using Iodobeads and following the manufacturer's protocol (Pierce Chemicals). Unless otherwise mentioned, all other reagents were obtained from Sigma Chemicals Co.

\section{Construction of pEG-1}

pGEMEX-1 was obtained from Promega and pEGFP-C1 from Clontech, and the EGFP gene was inserted into pGEMEX-1. Briefly, a 1619-nucleotide DNA fragment corresponding to a region of the pEGFP-C1 vector stretching from the CMV promoter to the SV40 polyadenylation site (1-1619 basepairs) was amplified by polymerase chain reaction using primers (Genemed Biotechnologies) containing an SfiI site in the $5^{\prime}$ and an SphI site in the $3^{\prime}$ overhang. The fragment and pGEMEX-1 were then digested with SphI and SfiI (New England Biolabs) and ligated to generate pEG-1. All plasmids were propagated in DH5 $\alpha$ cells and purified with a Plasmid Maxi Kit (Qiagen).

\section{Fluorescence Microscopy of Polyplex Gene Delivery}

Streptavidin-polylysine and biotin-EGF crosslinking and purification were conducted as previously described (Schaffer and Lauffenburger, 1998). Polylysines of average length 19, 36, and 180 residues (pK19, pK36, and pK180) were fluorescently labeled with Alexa 488 following the manufacturer's instructions (Molecular Probes). The degree of labeling was determined by using ninhydrin to quantify polylysine while measuring absorbance at $494 \mathrm{~nm}$ to determine dye content. pGEMEX-1 was biotinylated using psoralen-biotin reagent following the manufacturer's instructions (Ambion), and the DNA was separated from excess biotinylation reagent by dialysis vs. HBS using a 10,000MWCO membrane (Spectrum). One half milligram of streptavidin was labeled with Alexa 546 (Molecular Probes) and separated from free reagent by extensive dialysis vs. HBS using a 10,000-MWCO membrane. Biotinylated DNA was then incubated with a tenfold excess of fluorescently labeled streptavidin for $1 \mathrm{~h}$, and free biotin-binding sites were then blocked by addition of excess biotin. DNA was then separated from unlinked streptavidin and excess biotin by extensive dialysis vs. HBS using a 300,000-MWCO membrane (Spectrum).

To form polyplexes, fluorescently labeled plasmid was added to a $20 \mathrm{~m} M$ HEPES ( $\mathrm{pH} 7.0$ ) and $1 \mathrm{M} \mathrm{NaCl}$ solution at a concentration of $10 \mu \mathrm{g} / \mathrm{mL}$. Streptavidin-polylysine and fluorescently labeled polylysine were mixed at a lysine molar ratio of 1:4. This mixture was then added to the DNA solution at an electroneutral charge ratio while the solution was vortexed, and the resulting complexes were extensively dialyzed against HBS using a 1000-MWCO membrane (Spectrum). Loss of conjugate during dialysis was negligible. Biotinylated EGF was then added to the complexes at a valency, or ligand-to-plasmid ratio, of 15, and the solution was incubated for $1 \mathrm{~h}$.

Swiss 3T3-derived NR6 fibroblasts, which lack endogenous EGF receptors, were transduced with a gene encoding the wild-type human EGFR and selected with G418, as previously described (Chen et al., 1994). NR6 cells, maintained as described (Schaffer and Lauffenburger, 1998), were plated at 5000 cells $/ \mathrm{cm}^{2}$ on glass coverslips and grown overnight. Complexes were then added to cells in genetransfer medium, growth medium lacking FBS but instead containing $1 \%$ insulin-transferrin-selenium supplement (Life Technologies) plus $100 \mu M$ chloroquine. After a 6-h incubation, the conjugate solution was aspirated and replaced with regular growth medium lacking phenol red. After 48 h, samples were fixed with $4 \%$ paraformaldehyde, stained with $0.25 \mu \mathrm{g} / \mathrm{mL}$ DAPI (Molecular Probes) in PBS with $0.1 \%$ Triton, and mounted in Prolong Antifade reagent (Molecular Probes). Samples were visualized using a Nikon Eclipse E-800 fluorescence microscope with a 100× objective and a mercury arc lamp light source. A z-series of typically nine images with a spatial separation of $0.25 \mu \mathrm{m}$ was collected using a Photometrics AT200-cooled CCD camera controlled by CELLSCAN (Scanalytics) software, and deconvoluted using Exhaustive Photon Reassignment (Scanalytics) software. Samples lacking Alexa 488, Alexa 546 , or both labels were also visualized to analyze background levels of fluorescence.

\section{In Vitro Measurements of Conjugate Unpackaging}

The dissociation of polylysine of different lengths from plasmid DNA, due to cation exchange, was analyzed in vitro, a method similar to previous work (Ruponen et al., 1999; Zelphati and Szoka, 1996). Conjugates lacking fluorescent label were generated as described earlier, and in each sample approximately $50 \mathrm{ng}$ of DNA incorporated into 
conjugates at an electroneutral charge ratio was mixed with $10 \mu \mathrm{L}$ of double-stranded DNA immobilized to cellulose (Sigma) in a total volume of $60 \mu \mathrm{L}$ of HBS. Samples were agitated at $37^{\circ} \mathrm{C}$ for various times and centrifuged, and the supernatant was collected. Ten microliters of each sample were then run on a $1 \%$ agarose gel using TAE as buffer. Gels were stained with SYBR Gold (Molecular Probes) and visualized with a Fluor-S fluorescence imager (Bio-Rad Laboratories). Ten microliters of each of the $48 \mathrm{~h}$ samples were also run on the gel after complete dissociation of polylysine from the plasmid by the addition of $2 \mathrm{M} \mathrm{NaCl}$, and plasmid loss during the experiment was found to be negligible.

\section{In Vitro Transcription}

Polyplexes were generated essentially as described earlier using either pEG-1 or pGEMEX-1 and either streptavidinpolylysine or uncrosslinked polycations. Biotinylated EGF was also added to some complexes that contained streptavidin. Conjugates were extensively dialyzed into $10 \mathrm{mM}$ Tris buffer ( $\mathrm{pH} \mathrm{8.0)}$, and there was negligible loss during dialysis. Conjugates containing approximately $0.12 \mu \mathrm{g}$ of DNA were then added to a $30-\mu \mathrm{L}$ transcription reaction assembled using a T7 RNA Polymerase Kit (Ambion). For visualization, reactions and RNA molecular weight markers (Ambion) were electrophoresed on a formaldehyde agarose gel, stained with Radiant Red (Bio-Rad), and visualized with a Fluor-S fluorescence imager (Bio-Rad). For accurate quantitation of RNA, reactions containing $0.2 \mu \mathrm{Ci}$ of $\alpha 32 \mathrm{P}$ UTP (New England Nuclear) were run for $4 \mathrm{~h}$ at $37^{\circ} \mathrm{C}$, and RNA was separated from free mononucleotides using 30,000-MWCO Ultrafree-MC Centrifugal Filter Units (Millipore Corp.). After two washes with $0.5 \mathrm{~mL}$ of $\mathrm{HBS}$, the radioactivity retained on the filter was counted using a Packard Tri-Carb 2500 TR Liquid Scintillation Counter (Packard Instruments). In vitro transcriptions were also performed using polyethylenimine with averages of 46 and 600 monomers (Aldrich).

\section{Molecular Conjugate Gene Delivery}

For gene delivery experiments, 50,000 cells were seeded in 35-mm tissue culture dishes (Corning) and grown overnight. Polyplexes lacking any fluorescent label and containing either pEG-1 or pEGFP-C1 were generated at an electroneutral charge ratio as described earlier. Conjugates containing 0.38 pmol of plasmid DNA were then added to the cells in gene transfer medium. After a 6-h incubation, the conjugate solution was aspirated and replaced with regular growth medium. After $48 \mathrm{~h}$, the duplicate samples were trypsinized and analyzed for GFP expression using a FACScan flow cytometer (Becton Dickerson) with a 488$\mathrm{nm}$ excitation laser and a 530/30-nm fluorescein emission filter. Delivery was also conducted in the presence of 1 $\mu \mathrm{g} / \mathrm{mL}$ EGF to block binding of conjugates to the EGF receptor.

\section{Quantitation of Internalized Conjugate}

To quantify the internalization of conjugates composed of different polylysines, experiments were conducted with radiolabeled conjugates, as described elsewhere (Schaffer and Lauffenburger, 1998). Cells were seeded at 100,000 per $35-\mathrm{mm}$ plate and grown overnight. Briefly, 100,000 cpm of

${ }^{125} \mathrm{I}$-labeled SA-pK, iodinated to a specific activity of $65,000 \mathrm{cpm} / \mathrm{ng}$ of streptavidin were added for each microgram of $10 \mu \mathrm{g} / \mathrm{mL}$ pEG- 1 in $1 M \mathrm{NaCl}$ plus $20 \mathrm{~m} M$ HEPES while the solution was vortexed. Conjugates were then generated as described earlier, and $1 \mu \mathrm{g}$ of DNA in gene transfer medium with $100 \mu M$ chloroquine was added to cells for $6 \mathrm{~h}$. After removing conjugates bound to the cell surface and tissue culture plastic, the internalized radioactivity was counted with a Packard 5000 series gammacounter (Packard Instruments).

\section{RESULTS}

\section{Fluorescence Microscopy Analysis of Molecular Conjugate Delivery}

Both the polycation and DNA were fluorescently labeled in order to trace their subcellular localization during gene delivery. Polylysines were labeled with Alexa 488, resulting in the incorporation of one dye for every 40 to 50 lysine residues. After streptavidin was labeled at an Alexa 546 dye:protein ratio of 5.4, its complexation to pGEMEX plasmid DNA resulted in the incorporation 8.1 streptavidins per plasmid molecule. pGEMEX-1 was used for these experiments, because GFP expression from pEG-1 would interfere with fluorescence tracing of the conjugates.

After delivery of fluorescently labeled molecular conjugates to NR6 mouse fibroblasts, cells were fixed and stained with DAPI to localize the nucleus. Planar cell images were then generated by deconvolution of a z-series of fluorescence microscopy images. After incubation with conjugates generated with $\mathrm{pK} 180$, the polylysine and DNA were found to colocalize to a large extent in cytoplasmic vesicles of the endocytic network (Fig. 1) of every cell. In 8 of 100 cells examined, we also observed plasmid DNA within the nucleus, which was visualized by DAPI staining. In all such cells, pK180 colocalized with the nuclear plasmid DNA. Gene delivery was also analyzed after incubation of cells with conjugates composed of $\mathrm{pK} 36$, and the polycation and DNA were also found to colocalize in endosomal vesicles (Fig. 2). In contrast to pK180 conjugates, however, plasmid DNA observed in the cell nucleus did not colocalize with pK36 in any of the 10 of 100 cells with nuclearly localized plasmid. We found similar results with pK19. As controls, cell samples were also generated from conjugates lacking either or both the polylysine and DNA labels to measure background fluorescence; this background noise was found to be insignificant in all cases (data not shown). 

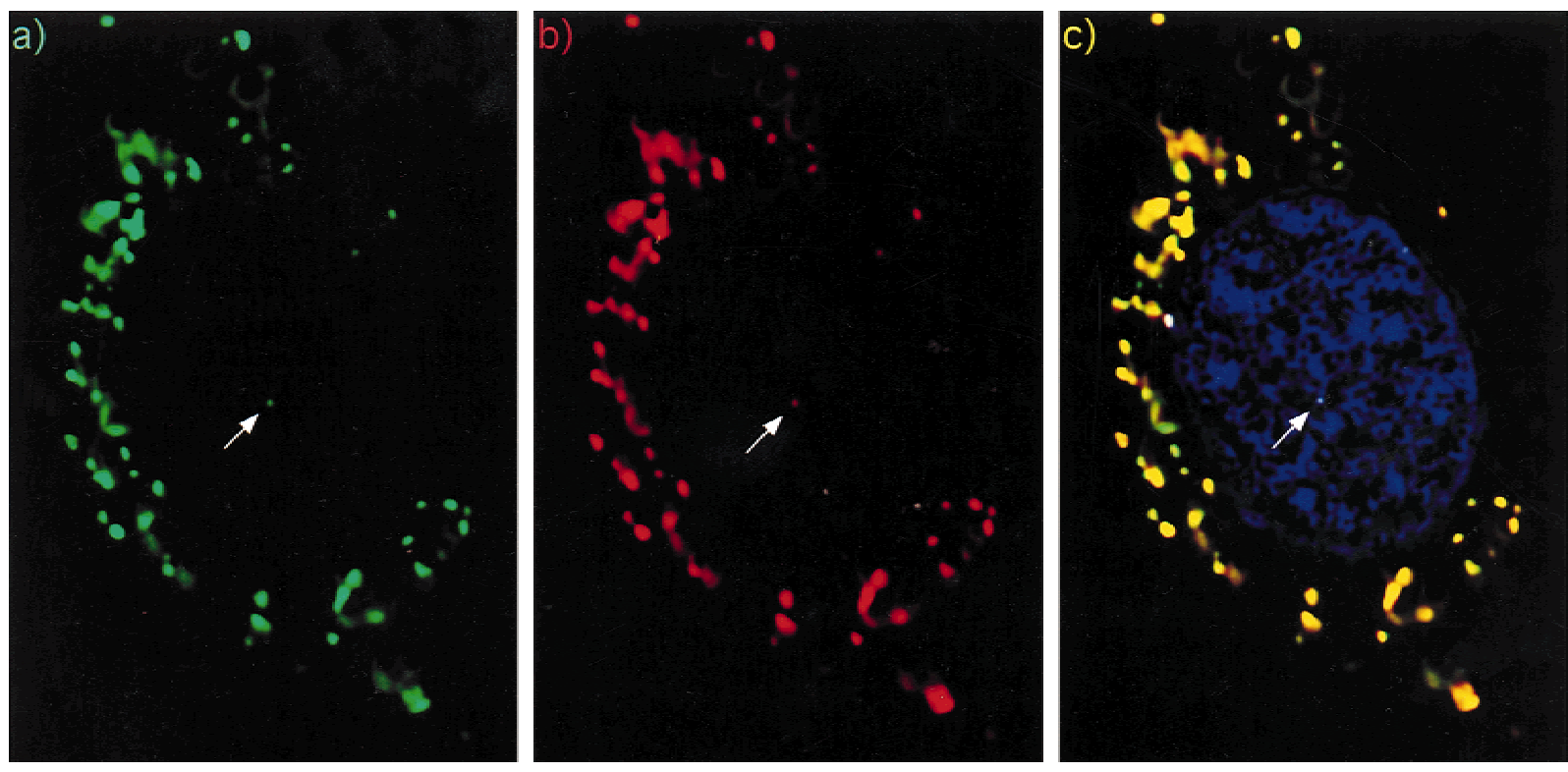

Figure 1. Representative planar fluorescent images of pK180 conjugate gene delivery. Fluorescently labeled conjugates were generated at an electroneutral charge ratio. (a) The green channel shows the localization of pK180 in NR6 cells after conjugate delivery, whereas (b) DNA localization is shown in red. (c) Colocalization is represented by yellow, and the nucleus is stained with DAPI (blue). Samples generated with conjugates lacking Alexa 488, Alexa 546, or both dyes demonstrated that background fluorescence was negligible.

\section{Construction of pEG-1}

Because the structure of polylysine-DNA complexes can depend on DNA sequence (Li et al., 1974; Shapiro et al., 1969; Weiskopf and Li, 1977), we generated one plasmid that could be used for all subsequent experiments in this study. The CMV promoter, GFP sequence, and SV40 polyadenylation site of pEGFP-N1 were inserted into pGEMEX-1 downstream of a T7 promoter and upstream of a T7 transcriptional terminator to generate the plasmid pEG-1. pEG-1 retains the ability direct expression of GFP in NR6 cells after transfection using LipofectAMINE (Life Technologies); however, it is less sensitive than pEGFP-C1, as it is expressed at lower levels as assayed by FACS (data not shown).

In addition to the CMV promoter and SV40 polyadenylation site, pEG-1 contains a T7 RNA polymerase promoter and transcriptional terminator; therefore, both T7 and RNA polymerase II transcription can proceed through the GFP coding region. This construct therefore permits the use of $\mathrm{T} 7 \mathrm{in}$ vitro transcription as a model for RNA synthesis in the cell nucleus. RNA transcribed from pEG-1 by T7 polymerase was electrophoresed on a formaldehyde agarose gel. Most transcripts were 2700 nucleotides in length, corresponding to RNA initiated at the promoter, encompassing the GFP and T7 gene 10, and ending at the T7 transcriptional terminator. A small fraction of elongations proceeded through the terminator to yield an approximately 8250nucleotide transcript after termination on the second pass (data not shown). In vitro transcription from the GFP coding sequence of DNA packaged into molecular conjugates can therefore be analyzed and quantified without having to lin- earize the plasmid and thereby potentially alter conjugate structure.

\section{In Vitro Measurement of Conjugate Unpackaging Rates}

The smaller polycation did not colocalize with plasmid DNA in the nucleus. Because this could potentially be due to rapid dissociation, or a lesser extent of association between polylysine and DNA, we measured the rates of conjugate dissociation in vitro. Conjugates were added to an approximately 800 -fold higher amount of double-stranded DNA immobilized to cellulose to mimic potential dissociation of polyplexes by chromosomal DNA. Samples were agitated for various times at $37^{\circ} \mathrm{C}$, separated from the excess DNA by centrifugation, and analyzed on an agarose gel. As shown in Figure 3, pK19 completely dissociated from the plasmid by $4 \mathrm{~h}$, and pK36 by $8 \mathrm{~h}$. In contrast, even after 48 h, pK180 was only approximately $50 \%$ dissociated from the plasmid DNA. The smaller polymers therefore released DNA more rapidly in vitro.

\section{In Vitro Transcription from Molecular Conjugate DNA}

We next investigated the potential consequences of the association between the polymer and DNA in the nucleus using in vitro transcription as a model for nuclear RNA synthesis. We found that pK180 significantly inhibited RNA synthesis, as shown in Figure 4. From a lysine:nucleotide ratio of 0 to 0.8 , transcription decreased slightly. However it dropped rapidly between a ratio of 0.8 and 1 and, for 

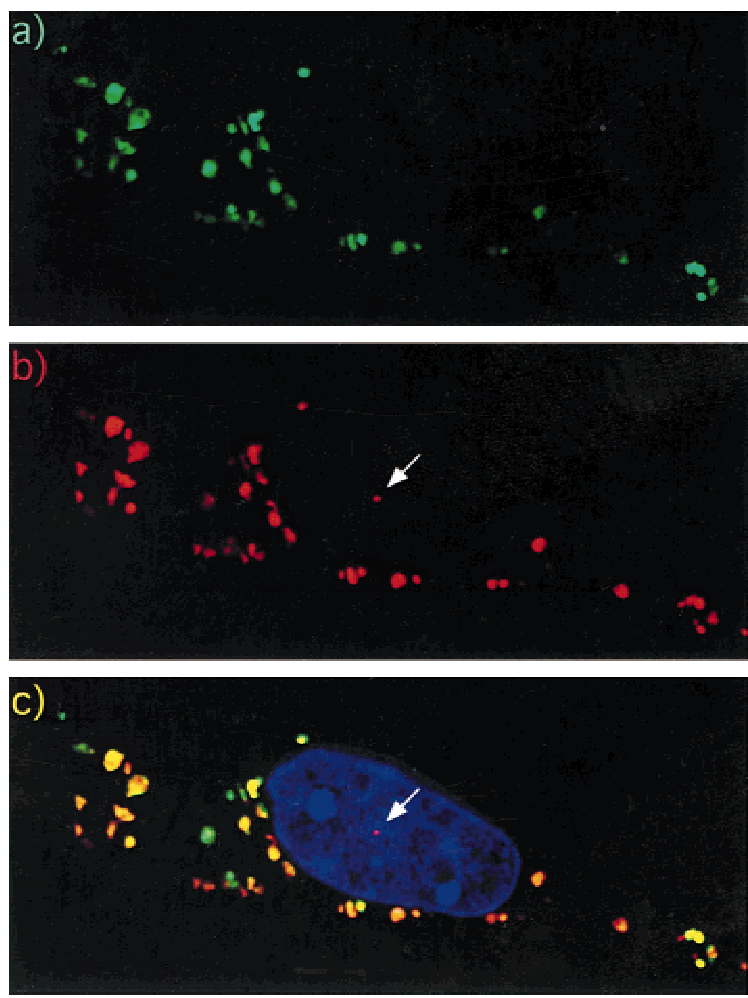

Figure 2. Representative planar fluorescent images of pK36 conjugate gene delivery. The polylysine (a) and plasmid DNA (b) colocalize to a large extent in endosomal vesicles (c). Unlike pK180, however, pK36 does not localize with the plasmid (c) in the nucleus. Results were similar for pK19.

all values $>1$, transcription levels were not statistically distinguishable from background $(P>0.05)$. The RNA transcripts generated were full length (data not shown), and thus the polylysine reduced the frequency of transcriptional initiation without greatly inhibiting elongation. Our results are consistent with the finding that both T7 and RNA polymerase II transcription from DNA condensed into polyplexes by cationic dendrimers are also reduced (Bielinska et al., 1997). In addition, complexation with polylysine was found to inhibit DNA replication in one report (Saffhill and Itzhaki, 1975).

There is evidence that the affinity between a polycation and polyanion depends on their size (Bakeev et al., 1992; Plank et al., 1999); therefore, the weaker binding of smaller polymers could potentially permit higher transcription rates in vitro. Accordingly, we assayed RNA synthesis from conjugates generated with polylysines of lengths 36 and 19 . Whereas the curve for pK36 overlapped with that of pK180 (Fig. 4), pK19 only reduced transcription to $60 \%$ even at charge ratios up to 5. Further addition of the polycation to a ratio of 15 decreased transcription only slightly. We also examined in vitro transcription from DNA complexed with another polycation, $\mathrm{PEI}$, and found that a $25-\mathrm{kDa}$ polymer inhibits transcription to a much greater extent than a $2-\mathrm{kDa}$ polycation (data not shown).

\section{Polyplex Gene Delivery}

Conjugates lacking any fluorescent label were incubated with cells for $6 \mathrm{~h}$, and gene delivery was quantified $48 \mathrm{~h}$ later by FACS analysis of GFP expression. Both pEG-1 and pEGFP-C1 were delivered. As shown in Table I, conjugates generated with pK36 showed the highest efficiency, up to $38 \%$, with pK19 slightly lower and and pK180 2.5-fold lower. Expression from pEGFP-C1 was detected in a higher fraction of cells, likely related to our aforementioned finding that it is more sensitive than pEG-1; however, the relative delivery efficiencies with the different polymers were the same with either plasmid. In addition, the delivery was receptor-mediated and dependent on polylysine, because the presence of saturating EGF, or a lack of polylysine (data not shown), significantly blocked expression. This difference in efficiency could arise from differences in the cellular uptake of conjugates generated with the three polylysines. To test this possibility, we measured conjugate internalization and found that a similar amount of conjugate was internalized regardless of polylysine length (Table I). We never observed nuclear localization of plasmid in greater than $10 \%$ of cells visualized by fluorescence microscopy, a low number compared with the observed gene delivery efficiencies. Thus, the microscopy may not have been sufficiently sensitive to detect all nuclear localization events. Alternatively, the fluorescent label may have partially interfered with conjugate delivery.

\section{Thermodynamic Model of Conjugate Dissociation}

There are several potential mechanisms for polyelectrolyte complex dissociation. First, the polycation and polyanion
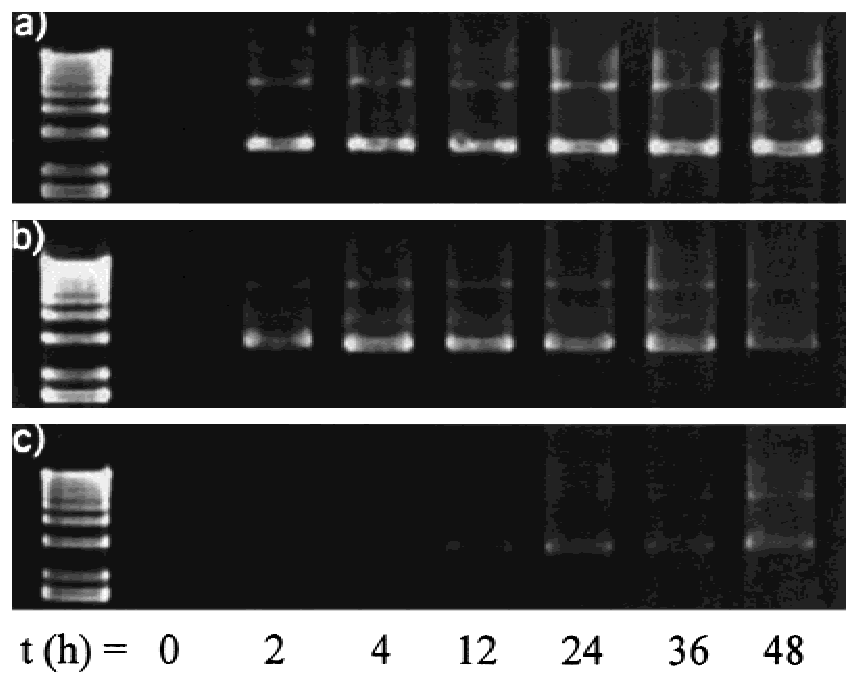

Figure 3. Dissociation of electroneutral charge ratio polyplexes by ion exchange of polylysine to excess DNA as a function of time (h). When polylysine dissociates from a plasmid, the DNA becomes free to migrate into an agarose gel. Whereas (a) pK19 releases all plasmid DNA within 4 $\mathrm{h}$ and (b) pK36 by $8 \mathrm{~h}$, even after $48 \mathrm{~h}$ (c) pK180 has only released approximately $50 \%$ of the plasmid DNA. 


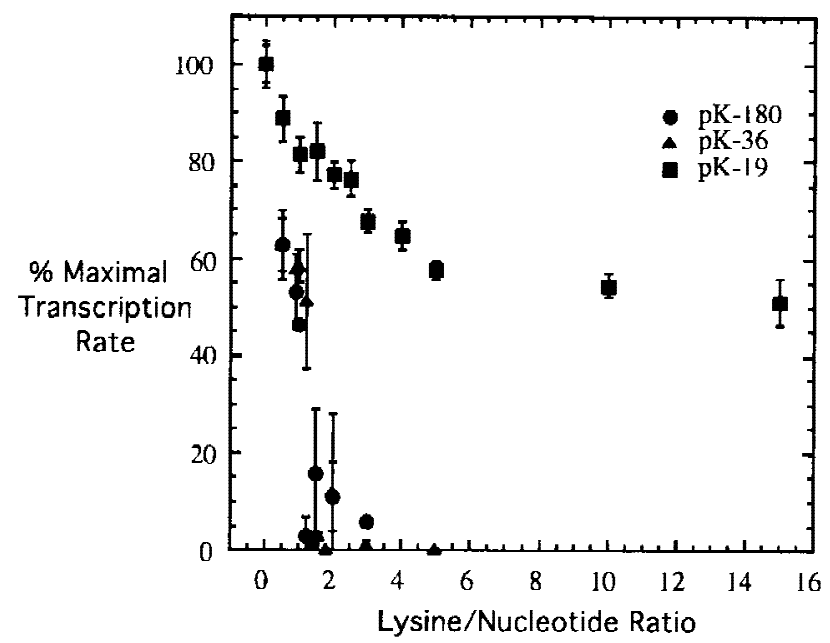

Figure 4. Polylysine inhibits RNA synthesis in vitro in a dose-dependent manner. RNA transcription in vitro from a T7 promoter was quantitated as a function of the dose and size of polylysine added to plasmid DNA. Data are reported as mean and standard deviation of two samples.

may spontaneously dissociate due to thermal energy. Alternatively, a third charged molecule can penetrate the complex and competitively dissociate one polymer from the other by ion exchange. We developed a simple thermodynamic model of the first mechanism to determine whether it can account for our observed dependency of molecular conjugate unpackaging on polylysine size (see Appendix for model development). By minimizing the free energy of a system composed of polycations of varying length adsorbed to a polyanion, an expression for the probability of complex dissociation, $P$, is obtained:

$$
P \sim e^{-\delta R} \frac{\delta}{\psi_{\psi}} R e^{-(1+\delta N)}
$$

where $N$ is the polymer chain length, $\delta$ is the bond energy between monomers of opposite charge, $R$ is the charge ratio, and $\psi_{0}$ is the polymer concentration. Therefore, for fixed polymer concentrations, the dissociation probability decreases with increasing polymer degree of polymerization $N$.

Table I. Gene delivery as a function of polylysine length. ${ }^{\mathrm{a}}$

\begin{tabular}{ccccc}
\hline $\begin{array}{c}\text { Polylysine } \\
\text { length }\end{array}$ & $\begin{array}{c}\text { pEG-1 } \\
\text { gene } \\
\text { delivery }\end{array}$ & $\begin{array}{c}\text { pEGFP-C1 } \\
\text { gene } \\
\text { delivery }\end{array}$ & $\begin{array}{c}\text { Delivery } \\
\text { with } \\
\text { excess EGF }\end{array}$ & $\begin{array}{c}\text { Internalized } \\
\text { plasmids/cell }\end{array}$ \\
\hline 19 & $16.9 \pm 1.1 \%$ & $32.5 \pm 2.5 \%$ & $1.9 \pm 0.05 \%$ & $110,700 \pm 6100$ \\
36 & $22.0 \pm 3.3 \%$ & $37.7 \pm 1.8 \%$ & $1.7 \pm 0.5 \%$ & $116,200 \pm 2800$ \\
180 & $7.5 \pm 0.8 \%$ & $15.3 \pm 0.6 \%$ & $1.8 \pm 0.3 \%$ & $124,900 \pm 5700$ \\
\hline
\end{tabular}

${ }^{a}$ NR6 fibroblasts were incubated with $1 \mu \mathrm{g}$ of either pEGFP-C1 or pEG-1 DNA, complexed with different polylysines at an electroneutral charge ratio, and assayed for GFP expression by FACS $48 \mathrm{~h}$ later. Delivery specificity was analyzed by including $1 \mu \mathrm{g} / \mathrm{mL}$ free EGF during the transfections. All data are reported as mean and standard deviation of three samples.

\section{DISCUSSION}

For in vivo polyplex gene delivery, the polycation condenses the DNA to protect it and facilitate its entry and passage through target cells. However, once inside the nucleus, in order to be processed by RNA transcription complexes, the DNA may first need to dissociate from the polycation. We have developed a system that employs a cationic polymer linked to epidermal growth factor as the ligand to deliver plasmid DNA encoding the green fluorescent protein to mouse fibroblasts (Schaffer and Lauffenburger, 1998; Schaffer et al., 1997). Here, we apply this system to investigate whether the unpackaging of a conjugate after delivery poses a barrier to gene expression.

In order to address this issue, we first developed fluorescent labeling and imaging methodology to analyze the gene transfer. The polycation, DNA, and cell nucleus were simultaneously labeled, and planar cell images were generated to localize them during the gene delivery process. We found that the polylysine and DNA remained largely colocalized in cytoplasmic vesicles. However, for conjugates that succeeded in escaping the endosome and localizing to the nucleus, the fate of the polycation depended on its degree of polymerization. pK180 remained colocalized with nuclear plasmid DNA. In contrast, we found no pK36 or pK19 in the vicinity of nuclear plasmid, and it is likely that these pKs completely dissociated from the plasmid, and therefore their signal was too diffuse to be observed. It is not known at what stage after internalization dissociation of the smaller polymer occurred, but the contrasting behavior may be due to differences in the affinities or the rates of dissociation of the polycations from the plasmid within the nucleus. To test this possibility, we incubated conjugates in the presence of a large excess of double-stranded DNA and found that $\mathrm{pK} 19$ and $\mathrm{pK} 36$ dissociated from the plasmid much more rapidly than $\mathrm{pK} 180$. This indicates that a slower rate of cation exchange of the polylysine to the surrounding chromatin could account for the continued association of pK180 with plasmid DNA observed in the microscopy results. Godbey et al. (1999a) also examined the trafficking of molecular conjugates, although the dimensions of the conjugate DNA we visualized in the nucleus were significantly smaller. However, the size we observed, approximately $\sim 100 \mathrm{~nm}$ or less was very consistent with light-scattering or electron-microscopy measurements of conjugate dimensions (Perales et al., 1997).

We next investigated the potential consequences of association between the polymer and DNA in the nucleus. We measured transcription from the $\mathrm{T} 7$ promoter of plasmid complexed with polylysine and found that the polycation significantly inhibited RNA synthesis. Transcription from DNA condensed at an electroneutral charge ratio with pK36, pK180, or PEI with 600 monomers was not above background. In contrast, inhibition by pK19 or PEI with 46 monomers was significantly less. Under the conditions of the in vitro dissociation experiment only pK180 conjugates did not unpackage for long periods of time; however, under 
the milder conditions of the transcription experiment, both pK180 and pK36 conjugates likely remained intact. The pK19 conjugates likely dissociated due to the presence of 2 $\mathrm{m} M$ spermidine in the in vitro transcription buffer, because this compound's three protonated amines may dissociate the smaller polymers from the DNA. Both spermidine and spermine are present intracellularly at concentrations in the millimolar range (Cohen, 1971; Tabor and Tabor, 1984); therefore, naturally occurring polyamines may play a role in dissociating polyplexes in addition to possible cation exchange of polylysine to chromatin. At any rate, these experiments imply that if polylysine does remain associated with the plasmid DNA, transcription is inhibited.

Because the larger polycation both remains associated with the plasmid in the nucleus at $48 \mathrm{~h}$ and reduces in vitro transcription, it would be predicted to lead to lower levels of gene expression. We found that, $48 \mathrm{~h}$ after gene delivery, GFP was expressed in $15 \%$ of cells using conjugates with pK180. With polyplexes generated with pK19 and pK36, however, GFP was expressed in $33 \%$ and $38 \%$ of target cells, respectively. This nearly threefold increase in the number of cells expressing a transgene is a significant improvement. These results also correlate well with the in vitro dissociation experiment, which showed that pK19 and pK36 conjugates rapidly dissociate in the presence of excess DNA. Because dissociation of pK180 from DNA appears to be slow, the difference in expression is likely caused by inhibition of gene expression due to plasmid association with pK180. Therefore, a vector may be successfully delivered to the nucleus, but a failure to uncoat can prevent the transgene from being expressed and detected.

We additionally developed a simple thermodynamic model to attempt to physically account for the differences observed both in vitro and in vivo in molecular conjugate dissociation as a function of polycation length. The model predicts that the probability of conjugate dissociation decreases with increasing polycation degree of polymerization. This effect is likely further enhanced by the presence of charged compounds in the cell, such as lipids, polyamines, or chromatin, that can potentially penetrate molecular conjugates and thereby competitively dissociate them. This behavior may be related to similar phenomena observed by Zelphati and Szoka with cationic liposomes (Zelphati and Szoka, 1996).

There is evidence that conjugate unpackaging may play a role in endosomal escape of DNA. For example, polycations that function as proton sponges likely release the DNA in the acidic endosomal network, and this dissociation has been proposed to be the mechanism for the ability of these polycations to enhance endosomal release (Boussif et al., 1995; Haensler and Szoka, 1993). In addition, neutralization of some of the positive charges of polylysine decreases its affinity for DNA and increases gene expression (Erbacher et al., 1997).

These studies conclude that partial or total conjugate dissociation facilitates endosomal escape; however, our results suggest that dissociation is also required for a later step in the gene transfer process, recognition, and efficient expression of delivered DNA by transcription machinery. Furthermore, consideration of the properties of the polycation may facilitate the design of polymers that more easily release their cargo once inside the nucleus. In addition to the polymer length, its structure, charge density, and biodegradability could influence its affinity for DNA. These polymer properties could also influence the duration of expression. For example, it is known that conjugate DNA can persist in endosomal vesicles, and our findings suggest that unpackaged conjugates can also exist in the nucleus (Edwards et al., 1996; Wu et al., 1995). It has recently been found that conjugates with shorter polycations have higher initial transgene expression, whereas longer polylysine conjugates yield more sustained expression (Ziady et al., 1999). Our results imply that this effect is due to the rapid release of plasmid DNA by the small polylysine, but a more sustained release of a plasmid reservoir by the longer polymer. Tuning the polymer properties can therefore afford control of not only the magnitude but also the duration of transgene expression.

Some reports have shown that short cationic polymers injected with plasmid DNA directly into a cell cytoplasm (Wolfert and Seymour, 1998) or into the cell nucleus (Pollard et al., 1998) do not appear to restrict transgene expression and, in fact, may even slightly enhance expression. The lack of restriction is consistent with our findings that shorter polycation molecules are able to unpackage their DNA cargo more efficiently than longer polymer vehicles, and an enhancement effect could be consistent with a protective reservoir mechanism. It is conceivable, in fact, that an intermediate degree of unpackaging proclivity-corresponding in this context to an intermediate polymer length-could yield optimal transgene expression.

Figure 5 illustrates the dependence of all our measured variables-including cell expression from both plasmid types and all three assay variables-on the polycation length, $N$. Clearly, none of the assay variables predicted cell expression entirely, as there was no reason to believe they should. The general trends of decreasing release and transcription as $N$ increases, along with the increasing colocalization of plasmid and polymer in the nucleus (plotted in terms of "noncolocalization" for easier trend comparison), were consistent with the decrease in gene expression efficiency for both plasmid types at high values of $N$. We also noted a mild optimum for expression efficiency at the intermediate polymer length, as suggested earlier. In any event, we believe that our combined battery of assays sheds important new light on the intricate dynamics of transgene delivery and expression by synthetic delivery vehicles.

The importance of viral uncoating in infection and replication is illustrated by the intricate and concerted process of virion disassembly, as exemplified by adenoviruses (Greber et al., 1993, 1994). Furthermore, a block in unpackaging reduces viral infection. For example, the parvovirus MVMi infects lymphocytes, but not fibroblasts, and its lack of replication in fibroblasts is apparently due to the inability of the 


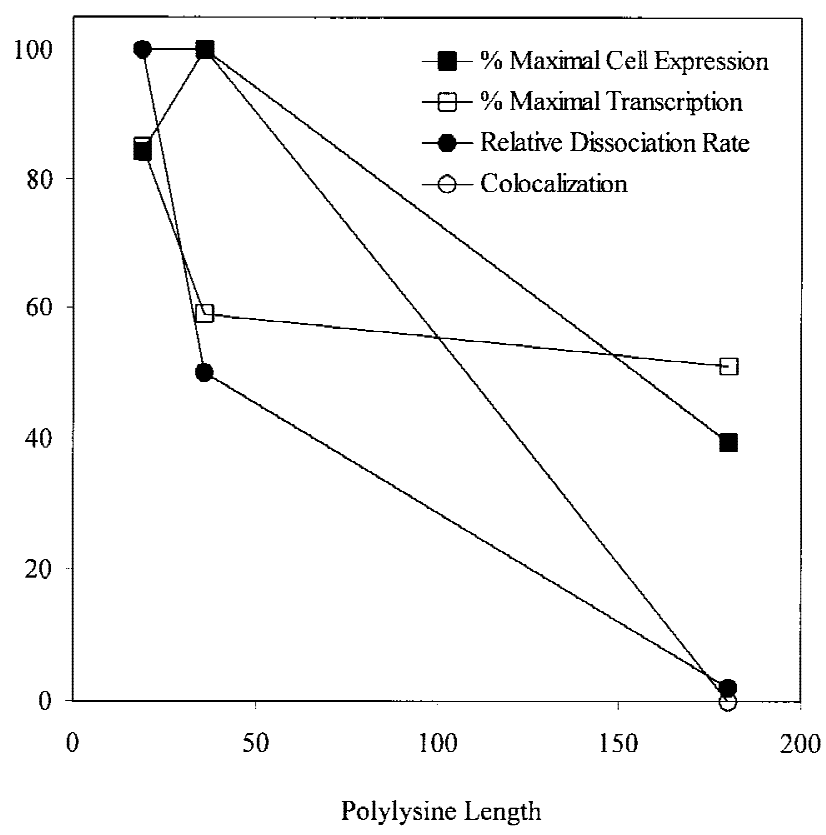

Figure 5. Dependence of measured variables on polylysine length, $N$. Expression from pEGFP-C1 (filled squares) is greatest for lower values of $N$, extent of in vitro transcription (open squares), competitive release (filled circles), and degree of plasmid/polymer noncolocalization in the nucleus (open circles) decrease as $N$ increases. In vitro transcription is taken from Figure 4 as the value at a polymer:plasmid ratio of unity. Competitive release is taken from Figure 3 as the time for $50 \%$ release, then normalized to the value for $N=19$. Noncolocalization is 1 minus the fraction of cells observed with plasmid and polymer colocalized in the nuclear region, from a population of ten cells for each polymer length.

capsid to uncoat in these cells. Also, the addition of neutralizing antibodies to poliovirus, influenza, vaccinia, or reovirus can inhibit uncoating and thereby prevent infection (Previsani et al., 1997; Rigg et al., 1989; Rodriguez et al., 1985; Virgin et al., 1994). Nature has realized that uncoating is a vital step in viral gene delivery and expression, and this efficient unpackaging is therefore one more lesson that synthetic vectors should learn from viruses.

\section{APPENDIX}

Here we outline a simple thermodynamic analysis of one of the possible explanatory mechanisms for the dependence of plasmid dissociation from cationic polymer on the polymer chain length, that of an equilibrium distribution between polymer complexed with DNA and free in solution. In this simple analysis, we idealize the complexation of flexible, positively charged polymer chains with negatively charged, rodlike DNA as an adsorption process.

Define the cationic polymer concentration in solution as $c_{p}$. The concentration of polymer chains is, therefore, $c_{p} / N$, where $N$ is the number of monomers per chain. This solution is then mixed with m DNA rods of length $L$, at a charge ratio $R$, which is proportional to $c_{p} / \mathrm{mL}$.

We define the fraction of polymer monomers in com- plexes with the DNA rods as $\phi$. Because the attraction between the rods and the polymers is strong and the chain concentration relatively low, we assume that the chains adsorb in a flat configuration. Therefore, the number of bonds formed in the complexes is approximately $\phi c_{p}$. If the energy per bond is defined as $\delta$ and the energy of a chain remaining free in solution is given by its chemical potential $\mu=1 \mathrm{n}(1$ $-\phi) c_{p} / N$, then the overall system energy is:

$$
F=-\delta \phi c_{p}+\frac{(1-\phi) c_{p}}{N} \ln \frac{(1-\phi) c_{p}}{N}
$$

All energies are given in units of $k T$, where $k$ is the Boltzmann coefficient and $T$ is the temperature. Minimization with respect to $\phi$ will yield the fraction of complexed monomers. If we assume that the complexed polymer chains are distributed uniformly between the DNA rods, we find that the number of polymer monomers per complex, $n_{c}$, given by $\phi c_{p} / m$, is proportional to:

$$
n_{c} \sim R\left(1-\frac{N}{c_{p}} e^{-(1+\delta N)}\right)
$$

We see that the number of polymer monomers bound per complex increases rapidly with chain molecular weight. The probability of a complex dissociating completely, $P$, is inversely proportional to the number of bonds. Using Boltzmann factor:

$$
P \sim e^{-\delta n_{c}} \sim \exp \left\{-\delta R\left(1-\frac{N}{c_{p}} e^{-(1+\delta N)}\right)\right\}
$$

Despite the simplicity of the model, this derivation provides several important insights. First, the charge ratio in the complex $\left(n_{c} / L\right)$ corresponds to the charge ratio in solution, $R$, only in the limit of high molecular weight. Furthermore, at fixed polymer concentration $c_{p}$, the probability of dissociation decreases rapidly either with the solution charge ratio $R$ or with the chain molecular weight $N$.

The model also predicts that if the complex charge is held fixed, the probability of dissociation should be independent of chain length. This is in agreement with a previous study that has shown that, for complexes of fixed stoichiometry, the dissociation is independent of chain length (Schindler and Nordmeier, 1997). Although the model does not consider exchange between the DNA rods and competing negatively charged macromolecules, the basic premise should hold.

The authors thank Rachael Neve for helpful technical discussions.

\section{References}

Bakeev KN, Izumrudov VA, Kuchanov SI, Zezin AB, Kabanov VA. 1992. Kinetics and mechanism of interpolyelectrolyte exchange and addition reactions. Macromolecules 25:4249-4254.

Bielinska AU, Kukowska-Latallo JF, Baker JR. 1997. The interaction of plasmid DNA with polyamidoamine dendrimers: mechanism of complex formation and analysis of alterations induced in nuclease sensi- 
tivity and transcriptional activity of the complexed DNA. Biochim Biophys Acta 1353:180-190.

Boussif O, Lezoualc'h F, Zanta MA, Mergny MD, Scherman D, Demeneix B, Behr J-P. 1995. A versatile vector for gene and oligonucleotide transfer into cells in culture and in vivo: polyethylenimine. Proc Natl Acad Sci USA 92:7297-7301.

Capecchi MR. 1980. High efficiency transformation by direct microinjection of DNA into cultured mammalian cells. Cell 22:479-485.

Chen P, Gupta K, Wells A. 1994. Cell movement elicited by the epidermal growth factor requires kinase and autophosphorylation but is separable from mitogenesis. J Cell Biol 124:547-555.

Chiou HC, Tangco MV, Levine SM, Robertson D, Kormis K, Wu CH, Wu GY. 1994. Enhanced resistance to nuclease degradation of nucleic acids complexed to asialoglycoprotein-polylysine carriers. Nuc Acids Res 22:5439-5446.

Cohen SS. Introduction to the polyamines. Englewood Cliffs, NJ: PrenticeHall; 1971.

Cotten M, Wagner E, Zatloukal K, Phillips S, Curiel DT, Birnstiel ML. 1992. High-efficiency receptor-mediated delivery of small and large (48 kilobase) gene constructs using the endosome-disruption activity of defective or chemically inactivated adenovirus particles. Proc Natl Acad Sci USA 89:6094-6098.

Curiel DT, Agarwal S, Wagner E, Cotten M. 1991. Adenovirus enhancement of transferrin-polylysine-mediated gene delivery. Proc Natl Acad Sci USA 88:8850-8854.

Edwards RJ, Carpenter DS, Minchin RF. 1996. Uptake and intracellular trafficking of asialoglycoprotein-polylysine-DNA complexes in isolated rat hepatocytes. Gene Ther 3:937-940.

Erbacher P, Roche AC, Monsigny M, Midoux P. 1997. The reduction of the positive charges of polylysine by partial gluconoylation increases the transfection efficiency of polylysine/DNA complexes. Biochim Biophys Acta 1324:27-36.

Godbey WT, Wu KK, Mikos AG. 1999a. Size matters: molecular weight affects the efficiency of poly(ethylenimine) as a gene delivery vehicle. J Biomed Mater Res 45:268-275.

Godbey WT, Wu KK, Mikos AG. 1999b. Tracking the intracellular path of poly(ethylenimine)/DNA complexes for gene delivery. Proc Natl Acad Sci USA 96:5177-5181.

Greber UF, Singh I, Helenius A. 1994. Mechanisms of virus uncoating. Trends Microbiol 2:52-56.

Greber UF, Willetts M, Webster P, Helenius A. 1993. Stepwise dismantling of adenovirus 2 during entry into cells. Cell 75:477-486.

Haensler J, Szoka FC. 1993. Polyamidoamine cascade polymers mediate efficient transfection of cells in culture. Bioconj Chem 4:372-379.

Hart SL, Harbottle RP, Cooper R, Miller A, Williamson R, Coutelle C. 1995. Gene delivery and expression mediated by an integrin-binding peptide. Gene Ther 2:552-554.

Kovesdi I, Brough DE, Bruder JT, Wickham TJ. 1997. Adenoviral vectors for gene transfer. Curr Opin Biotechnol 8:583-589.

Li HJ, Brand B, Rotter A. 1974. Thermal denaturation of calf thymus DNA: existence of a GC-richer fraction. Nucl Acids Res 1:257-265.

Paillard F. 1998. Oligoplexes: nonviral vehicles for receptor-mediated delivery. Hum Gene Ther 9:987-988.

Perales JC, Grossmann GA, Molas M, Liu G, Ferkol T, Harpst J, Oda H, Hanson RW. 1997. Biochemical and functional characterization of DNA complexes capable of targeting genes to hepatocytes via the asialoglycoprotein receptor. J Biol Chem 272:7398-7407.

Plank C, Mechtler K, Szoka FC, Wagner E. 1996. Activation of the complement system by synthetic DNA complexes: a potential barrier for intravenous gene delivery. Hum Gene Ther 7:1437-1446.

Plank C, Oberhauser B, Mechtler K, Koch C, Wagner E. 1994. The influence of endosome-disruptive peptides on gene transfer using synthetic virus-like gene transfer systems. J Biol Chem 269:12918-12924.

Plank C, Tang MX, Wolfe AR, Szoka FC. 1999. Branched cationic peptides for gene delivery: role and type and number of cationic residues in formation and in vitro activity of DNA polyplexes. Hum Gene Ther 10:319-332.

Pollard H, Remy J-S, Loussouarn G, Demolombe S, Behr J-P, Escande D.
1998. Polyethylenimine but not cationic lipids promotes transgene delivery to the nucleus in mammalian cells. J Biol Chem 273: $7507-7511$.

Previsani N, Fontana S, Hirt B, Beard P. 1997. Growth of the parvovirus minute virus of mice MVMp3 in EL4 lymphocytes is restricted after cell entry and before viral DNA amplification: cell-specific differences in virus uncoating in vitro. J Virol 71:7769-7780.

Rigg RJ, Carver AS, Dimmock NJ. 1989. IgG-neutralized influenza virus undergoes primary, but not secondary uncoating in vivo. J Gen Virol 70:2097-2109.

Rodriguez JF, Janeczko R, Esteban M. 1985. Isolation and characterization of neutralizing monoclonal antibodies to vaccinia virus. J Virol 56: $482-488$.

Ruponen M, Yla-Herttuala S, Urtti A. 1999. Interactions of polymeric and liposomal gene delivery systems with extracellular glycosaminoglycans: physiochemical and transfection studies. Biochem Biophys Acta 1415:331-341.

Saffhill R, Itzhaki RF. 1975. Accessibility of chromatin to DNA polymerase I and location of the F1 histone. Nucl Acids Res 2:113-119.

Schaffer DV, Lauffenburger DA. 1998. Optimization of cell surface binding enhances efficiency and specificity of molecular conjugate gene delivery. J Biol Chem 273:28004-28009.

Schaffer DV, Neve RL, Lauffenburger DA. 1997. Use of the green fluorescent protein as a quantitative reporter of epidermal growth factor receptor-mediated gene delivery. Tissue Eng 3:53-63.

Schindler T, Nordmeier E. 1997. The stability of polyelectrolyte complexes of calf-thymus DNA and synthetic polycations. Macromol Chem Phys 198:1943-1972.

Sebestyen MG, Ludtke JJ, Bassik MC, Zhang G, Budker V, Lukhtanov EA, Hagstrom JE, Wolff JA. 1998. DNA vector chemistry: the covalent attachment of signal peptides to plasmid DNA. Nature Biotechnol 16:80-85.

Shapiro JT, Leng M, Felsenfield G. 1969. Deoxyribonucleic acid-polylysine complexes. Structure and nucleotide specificity. Biochemistry 8: 3219-3232.

Sosnowski BA, Gonzalez AM, Chandler LA, Buechler YJ, Pierce GF, Baird A. 1996. Targeting DNA to cells with basic fibroblast growth factor (FGF2). J Biol Chem 271:33647-33653.

Stankovics J, Crane AM, Andrews E, Wu CH, Wu GY, Ledley FD. 1994. Overexpression of human methylmalonyl CoA mutase in mice after in vivo gene transfer with asialoglycoprotein/polylysine/DNA complexes. Hum Gene Ther 5:1095-1104.

Tabor CW, Tabor H. 1984. Polyamines. Annu Rev Biochem 53:749-790.

Virgin HW, Mann MA, Tyler KL. 1994. Protective antibodies inhibit reovirus internalization and uncoating by intracellular proteases. J Virol 68:6719-6729.

Wagner E, Curiel D, Cotten M. 1994. Delivery of drugs, proteins and genes into cells using transferrin as a ligand for receptor-mediated endocytosis. Adv Drug Del Rev 14:113-135.

Weiskopf M, Li HJ. 1977. Poly(L-lysine)-DNA interactions in $\mathrm{NaCl}$ solutions: $\mathrm{B} \rightarrow \mathrm{C}$ and $\mathrm{B} \rightarrow$ Psi transitions. Biopolymers 16:669-684.

Wolfert MA, Seymour LW. 1998. Chloroquine and amphipathic peptide helices show synergistic transfection in vitro. Gene Ther 5:409-414.

Wu GW, Chowdhury JR, Bommineni VR, Basu SK, Wu CH, Chowdhury NR. 1995. Fate of DNA targeted to hepatocytes by asialoglycoprotein polylysine conjugates. Proc Assoc Am Phys 107:211-217.

Wu GY, Wu CH. 1987. Receptor-mediated in vitro gene transformation by a soluble DNA carrier system. J Biol Chem 262:4429-4432.

Zelphati O, Szoka FC Jr. 1996. Mechanism of oligonucleotide release from cationic liposomes. Proc Natl Acad Sci USA 93:11493-11498.

Ziady A-G, Ferkol T, Dawson DV, Perlmutter DH, Davis PB. 1999. Chain length of the polylysine in receptor-targeted gene transfer complexes affects duration of reporter gene expression vitro and in vivo. J Biol Chem 274:4908-4916.

Ziady AG, Perales JC, Ferkol T, Gerken T, Beegen H, Perlmutter DH, Davis PB. 1997. Gene transfer into hepatoma cell lines via the serpin enzyme complex receptor. Am J Physiol 273:G545-G552. 